Simulation of spray dispersion in a simplified heavy vehicle wake

J.S. Paschkewitz

January 17, 2006 
This document was prepared as an account of work sponsored by an agency of the United States Government. Neither the United States Government nor the University of California nor any of their employees, makes any warranty, express or implied, or assumes any legal liability or responsibility for the accuracy, completeness, or usefulness of any information, apparatus, product, or process disclosed, or represents that its use would not infringe privately owned rights. Reference herein to any specific commercial product, process, or service by trade name, trademark, manufacturer, or otherwise, does not necessarily constitute or imply its endorsement, recommendation, or favoring by the United States Government or the University of California. The views and opinions of authors expressed herein do not necessarily state or reflect those of the United States Government or the University of California, and shall not be used for advertising or product endorsement purposes.

This work was performed under the auspices of the U.S. Department of Energy by University of California, Lawrence Livermore National Laboratory under Contract W-7405-Eng-48. 


\title{
Simulation of spray dispersion in a simplified heavy vehicle wake
}

\author{
By J OHN S. PASCHKEWITZ \\ Energy and Environment Directorate, Lawrence Livermore National Laboratory, Livermore,
} CA, 94551

13 January 2006

Simulations of spray dispersion in a simplified tractor-trailer wake have been completed with the goal of obtaining a better understanding of how to mitigate this safety hazard. The Generic Conventional Model (GCM) for the tractor-trailer was used. The impact of aerodynamic drag reduction devices, specifically trailer-mounted base flaps, on the transport of spray in the vehicle wake was considered using the GCM. This analysis demonstrated that base flaps including a bottom plate may actually worsen motorist visibity because of the interaction of fine spray with large vortex flows in the wake. This work suggests that to use computational fluid dynamics (CFD) to design and evaluate spray mitigation strategies the jet or sheet breakup processes can be modeled using an array of injectors of small $(<0.1 \mathrm{~mm})$ water droplets; however the choice of size distribution, injection locations, directions and velocities is largely unknown and requires further study. Possible containment strategies would include using flow structures to "focus" particles into regions away from passing cars or surface treatments to capture small drops.

\section{Introduction}

Splash and spray around heavy trucks is a longstanding safety problem and has been investigated numerous times over the last thirty years (Weir et al. 1978; Manser et al. 2003; NHTSA 2000; Allan \& Lilley 1983). We define splash as the displacement of water in puddles by the tires both toward and outward from the truck and spray as very small droplets or mist resulting from the impact of splash or rain on truck or tire surfaces or from aerodynamic or collisional breakup of droplets ejected from the tire treads. The statistical evidence for splash and spray being a significant safety hazard is weak: approximately 20 out of 20,000 accidents were attributed to this problem in the last National Highway Traffic Safety Administration (NHTSA) report to Congress (NHTSA 2000). However, as stated in the NHTSA report (NHTSA 2000), it is possible that accidents due to splash and spray may be attributed to other causes such as driver error and slippery conditions, leading to a underestimate of the problem. Other reports suggest that public perception is that splash and spray is a major problem. For example, the American Automobile Association (AAA) claims that truck splash and spray is one of the most frequent safety complaints reported by motorists (NHTSA 2000; Manser et al. 2003). In response to public opinion, both the state of Oregon (O.D.O.T. 2002) and the European Union have mandated that trucks utilize some form of splash and spray control. There are a wide variety of aftermarket add-on devices that claim to mitigate the problem, but the experimental test record (Dumas \& Lemay 2004; Manser et al. 2003; Weir et al. 1978; Allan \& Lilley 1983; Goering \& Kramer 1987b,a) is rather mixed. Some studies claim 
that particular fairings or devices are effective while others show the same devices have no measurable impact. Some of the inconsistency in the experimental data reflects the considerable difficulties in obtaining repeatable quantitative measurements of the spray cloud around the truck. Dumas \& Lemay (2004) employed SAE recommended practice J2245, which reflects the most current attempt to define a rigorous road test protocol for measuring the spray cloud. This method uses laser-based line-of-sight opacity measurements along the sides of the truck, and Dumas \& Lemay (2004) found that even modest crosswinds created large disparities in the data. Given the considerable expense and uncertainty associated with these full-scale tests, it is highly desirable to develop computational approaches to provide insight into whether or not an add-on device is effective.

The impact of truck and tire aerodynamics on spray cloud formation and transport is less ambiguous than that of any aftermarket add-on devices. The experimental studies of Goering \& Kramer $(1987 a, b)$ illustrated that both splash and spray are strongly mitigated by using fully faired wheels. Fully faired wheels are not an operationally acceptable solution since brake cooling and mud or snow fouling are negatively impacted, but these studies clearly illustrate that improving tire aerodynamics has a direct impact on spray transport. Manser et al. (2003) examined the effectiveness of a variety of spray suppression add-on devices using both a 1985 and 1997 Freightliner tractor. None of the add-on devices were shown to have a statistically meaningful impact on the spray cloud, but the more streamlined tractor had measurably smaller spray cloud. This result is unsurprising since the width of the spray plume downstream of the truck is heavily dependent on the "bow wave" generated by the tractor and a more streamlined nose will decrease this width.

It is less clear that drag reducing add-on devices, in particular trailer base flaps, will have a similarly positive effect. Both CFD and experimental studies of truck-like geometries using base flaps have shown that the wake structure is modified. Spray droplets can interact with resulting vortex structures in the flow: for example, the well-known "particle focusing" effect (Tang et al. 1992, e.g.) in which small, low inertia particles are trapped in vortex cores while larger particles with finite inertia are concentrated along vortex edges. These finite inertia particle effects may actually act to decrease motorist visibility and determining if base flaps with a bottom plate actually reduce motorist visibility was one of the objectives of this study.

The experimental study of Manser et al. (2003) describes the current hypothesis for the origin of much of the spray cloud: spray results from droplet impacts with the truck undercarriage and the resulting fine mist is convected into the truck wake. Manser et al. (2003) claimed that the use of absorbent full-length mudflaps (again, unacceptable for snowy or muddy conditions) or undercarriage treatments combined with the use of a more aerodynamic tractor "definitely helped reduce spray cloud density." Based on this claim, a second set of objectives of this study was to determine the approximate size of droplets in the spray cloud and to investigate the transport of droplets in the tractortrailer wake, as well as to obtain some understanding of spray generation mechanisms. Specifically, do droplets break up because of aerodynamic stresses during their rather limited time-of-flight or do small drops largely result from collisions with truck or tire surfaces? We will demonstrate that the latter is the dominant effect.

In this report, simulation results obtained using the commercial computational fluid dynamics (CFD) solver StarCD (cd adapco 2005) are presented for spray propagation in a simplified heavy vehicle wake. In Section 2, the underlying equations and modeling assumptions used in the spray calculations are presented in addition to the StarCD simulation parameters are discussed. The work presented here has utilized an unsteady 
Reynolds-Averaged Navier-Stokes (URANS) turbulence modeling approach combined with empirical correlations for droplet behavior including aerodynamic and collisioninduced breakup. In Section 3, simulation results for the truck Generic Conventional Model (GCM) geometry are presented both with and without trailer base flaps. In the last section, our findings are summarized and directions for future research into this problem are discussed.

\section{Problem formulation}

\subsection{Governing equations for fluid flow}

We utilize the well-known Reynolds-Averaged Navier-Stokes (RANS) approach in a timedependent manner. The velocity field is decomposed into mean and fluctuating components Eq.2.1; the resulting ensemble-averaged Navier-Stokes equations Eq.2.2 contain an unknown Reynolds stress term that must be defined using a closure approximation.

$$
\begin{gathered}
u_{i}\left(x_{k}, t\right)=U_{i}\left(x_{k}\right)+u^{\prime}\left(x_{k}, t\right) \\
\frac{D U_{i}}{D t}=-\frac{1}{\rho} \frac{\partial P}{\partial x_{i}}+\frac{\partial}{\partial x_{j}}\left(\nu \frac{\partial U_{i}}{\partial x_{j}}\right)+\frac{\partial\left(\overline{-u_{i}^{\prime} u_{j}^{\prime}}\right)}{\partial x_{j}}
\end{gathered}
$$

In this work we use the Menter Shear Stress Transport (SST) turbulence model (Menter 1994) to model the Reynolds stresses. This model was chosen since it is implemented in StarCD and has been shown in previous heavy vehicle aerodynamics CFD studies (Salari et al. 2004a) to be more accurate than the simple $k-\epsilon$ and $k-\omega$ models. This twoequation model is based on the Boussinesq hypothesis that the Reynolds stresses are proportional to the local rate of strain, with the proportionality being the eddy viscosity $\nu_{T}$ :

$$
\overline{-u_{i}^{\prime} u_{j}^{\prime}}=2 \nu_{T} S_{i j}
$$

The SST model relates the eddy viscosity to the turbulent kinetic energy $\mathrm{k}$ and the turbulence frequency $\omega$ :

$$
\nu_{T}=C_{\mu} k^{2} / \omega
$$

Transport equations are solved for both $\mathrm{k}$ and $\omega$. By using a weighted average of the $k-\epsilon$ and $k-\omega$ models, with $k-\epsilon$ in the far-field and $k-\omega$ near the wall, the SST model obtains the superior behavior of the $k-\omega$ model near boundaries and for flows with streamwise pressure gradients. This improved performance is obtained while also avoiding the sensitivity of the $k-\omega$ model to the free-stream boundary condition on $\omega$. The default values of the model constants in StarCD correspond to those in the work of Menter (1994).

As is common in commercial code RANS implementations, the "high Reynolds number" turbulence model is coupled to a wall model to compensate for the lack of near-wall resolution in the computational mesh. In the zone below the first grid point away from the wall, the turbulence equations are abandoned and the turbulence and velocity are assumed to follow specified profiles. The wall function thus acts as a boundary condition for the turbulence model at the first grid point off the wall. The wall function approach makes a number of important assumptions: velocity variations are predominantly 1-D and normal to the wall, pressure gradients are negligible and that turbulence production and dissipation are balanced in the wall function zone. Shear stress and velocity are assumed to be aligned and unidirectional in the near-wall region. Given a solution for the Reynolds-averaged velocity, the skin friction is obtained from the empirical "law of the wall"; the skin friction is then used to obtain a boundary value for $\mathrm{k}$ (and $\epsilon$ or $\omega$ 
as appropriate). Additional details on wall functions are provided in Wilcox (2002) and Durbin \& Reif (2003).

For the results presented here, we have used the wall function approach. As these flows have a significant amount of separation, strong curvature, and adverse pressure gradients, we do not anticipate that the resulting wall shear stress predictions will be quantitatively accurate. However, for the preliminary studies performed here that are largely focused on the transport of spray by large-scale flow structures away from the boundaries, we anticipate that our results are not substantially affected by the use of these wall functions.

\subsection{Governing equations for droplet motion}

The droplet motion and breakup are calculated in the Lagrangian frame of reference. This approach requires interpolation of the surrounding carrier fluid velocity onto the centerof-mass of the computational particle representing the droplet. As is common practice in commerical CFD solvers, StarCD uses a "parcel" approach in which each particle represents a collection of droplets with a fixed mass. If the droplets are broken or coalesce, the number of parcels does not change; instead the number of droplets represented by the parcel is modified. The one exception to this rule in StarCD is discussed in Section 2.3. The parcel methodology keep the number of particles required to simulate the droplet physics manageable as explicit simulation of the millions of droplets present in even modest atomization problems is not possible due to the enormous computational cost. However, the parcel methodology also underestimates the resulting dispersion of a spray cloud. Lacking a better means of addressing this shortcoming, we used as large a number of parcels as computationally manageable.

The droplet motion in the Lagrangian frame of reference is described by:

$$
m_{d} \frac{d \vec{U}_{d}}{d t}=\vec{F}_{d}+\vec{F}_{p}+\overrightarrow{F_{v m}}+\vec{F}_{b}
$$

The aerodynamic drag $\vec{F}_{d}$ is proportional to the slip velocity of the droplets (which are assumed to be perfectly spherical):

$$
\overrightarrow{F_{d}}=\frac{1}{2} C_{d} \rho_{p} A_{p}\left|\vec{u}-\overrightarrow{u_{p}}\right|\left(\vec{u}-\overrightarrow{u_{p}}\right) .
$$

The effect of local pressure gradients on the particle or droplet motion is given by:

$$
\overrightarrow{F_{p}}=-V_{d} \nabla p .
$$

In this work, we have chosen to neglect the forces due to "virtual mass" $\left(F_{v m}\right)$, which results from the work required to displace the carrier fluid displaced by the drop and buoyancy forces $\left(F_{b}\right)$; scoping simulations in a crossflow atomizer and plane wake including these effects showed minimal differences.

Finally, the backcoupling of particle stresses on the flow field calculation ("two-way" coupling) was not included in this preliminary investigation. The importance of back-

coupling can be estimated using a momentum coupling parameter, which is the ratio of particle drag to the carrier fluid momentum flux:

$$
\Pi \approx \frac{C}{1+S t},
$$

where $C$ is the ratio of the mass flow rates of the disperse and continuous phases and $S t$ is the Stokes number, which is the ratio of the response times of the disperse and continuous 
phases and quantifies the relative importance of particle (disperse phase) inertia:

$$
S t=\frac{\rho_{p} d_{p}^{2} U}{18 \mu_{c} H},
$$

where the $p$ and $c$ subscripts denote the particle and continous phases, $\rho$ is the density, $d_{p}$ is the particle or droplet diameter, $U / H$ is a characteristic flow time scale, and $m u_{c}$ is the dynamic viscosity Crowe et al. (1998). If this parameter is greater than unity, the backcoupling of particle or droplet momentum on the carrier flow field may be important. A simple estimate of the importance of the coupling parameter is obtained as follows. Assuming an air flow rate of $20 \mathrm{~m} / \mathrm{s}$ and a $0.25 \mathrm{~m}^{2}$ frontal area near the tire, the mass flow rate is approximately $5 \mathrm{~kg} / \mathrm{s}$. The amount of water displaced by the tire patch can be estimated using a tire width $(50 \mathrm{~cm})$ multiplied by a tangential velocity $(20 \mathrm{~m} / \mathrm{s})$ multiplied by a water film thickness $(1 \mathrm{~mm})$ to give a mass flow rate of $10 \mathrm{~kg} / \mathrm{s}$. This gives a mass loading ratio of 2 in the immediate vicinity of the tire. For droplets with a diameter of $0.1 \mathrm{~mm}$, the Stokes number is 0.6 using the ratio of the free stream velocity and tire height $(1 \mathrm{~m})$ as the characteristic velocity. These parameters give $\Pi=1.25$, which suggests that backcoupling is important near the tires and for small (low St) droplets. As the spray disperses away from the tires, the mass loadings of water strongly decrease and coupling can be ignored; similarly, for very large droplets near the tires, there is little interaction between the droplets and the flow. Based on these estimates, the smaller droplet behavior near the tires would be modified if backcoupling were included but that the transport of droplets away from the near tire region is accurately modeled without these effects included.

\subsection{Empirical relationships for droplet physics}

Modeling the various processes of droplet breakup, particularly from unstable liquid sheets or jets, is extremely challenging and an active research area. The reviews of Lasheras \& Hopfinger (2000); Lin \& Reitz (1998); Lasheras et al. (2002); Kolev (1993) discuss the physical processes involved in jet breakup and droplet formation as well as statistical models describing these processes. Accounting for the physics of droplet impact with wetted or dry surfaces or interdroplet collisions is also computationally challenging and representative fundamental numerical studies are presented in Tsouris \& Tavlarides (1994); Sundaram \& Collins (1997); Chen et al. (1998); Josserand \& Zaleski (2003); Bussman et al. (1999). Although volume-of-fluid (VOF) (Scardovelli \& Zaleski 1999) or level set (Sethian \& Smereka 2003) methods can be used to examine the breakup of individual droplets with finite inertia, these approaches are computationally intractable for large number of droplets. As such, we are reduced to using empirical approaches to model the droplet formation, breakup and collision physics. These relationships are generally wellaccepted within the combustion community and are implemented in most commercial CFD codes. We do not anticipate that these relationships will be quantitatively accurate for the water sprays considered here but they should provide qualitative estimates of behavior.

In this work, we use the most "advanced" empirical models available in StarCD. For droplet breakup, we use the model of Pilch \& Erdman (1987). This model relates experimental observations of various droplet breakup modes to the Weber number, defined as:

$$
W e=\frac{\rho V^{2} D}{\sigma},
$$

where $\rho$ is the carrier fluid density, $V$ is the slip velocity between the droplet and the carrier fluid, $D$ is the drop diameter, and $\sigma$ is the surface tension of the droplet. The 
Weber number is a ratio of the inertial force, which acts to break the drop, to the stabilizing surface tension force. The second important parameter in the model is the Ohnesorge number:

$$
O h=\frac{\mu_{d}}{\sqrt{\rho_{d} D \sigma}},
$$

where $\mu_{d}$ is the dynamic viscosity of the droplet liquid, and $\rho_{d}$ is the droplet density. This parameter is a measure of the importance of droplet fluid viscous to surface tension forces and assumes that the carrier fluid viscosity is small compared to that of the droplet. Pilch \& Erdman (1987) curve-fitted data for various breakup modes to ranges of Weber number to derive their model, which allows one to obtain a characteristic time scale for breakup as well as an estimate for the largest "stable" drop diameter at which no further breakup occurs. Droplet breakup occurs if the Weber number exceeds a "critical" value based on a correlation of experimental data:

$$
W e_{c}=12\left(1+1.077(\mathrm{Oh})^{1.6}\right)
$$

If the critical Weber number is exceeded for a given droplet, a dimensionless breakup time, T, based on experimental correlations is obtained and are summarized in Table 1. These correlations assume that the droplet fluid has a low viscosity or $O h<0.1$; for water and droplets having a diameter of $1 \times 10^{-3}$ meters, $O h \approx 4 \times 10^{-3}$. The breakup time is non-dimensionalized by the ratio $D /\left(V \epsilon^{0.5}\right)$, where $\epsilon$ is the ratio of the continuous to disperse phase densities. This ratio is a time scale obtained using the relative inertia of the two fluids.

The dynamics of drop breakup are then accounted for with several empirical relationships. The maximum stable droplet diameter, $D_{m}$, is estimated by noting that the largest stable diameter is the one at which the Weber number equals the critical Weber number with a correction for the fact that as the droplet breaks, the droplet velocity decreases. This correction uses an estimate for the velocity of the droplet "cloud" that results post-breakup:

$$
D_{m}=W e_{c} \frac{\sigma}{\rho V^{2}}\left(1-\frac{V}{V_{d}}\right)^{-2},
$$

where $V_{d}$ is the post-breakup velocity of the droplet cloud. This velocity is obtained using another empirical relationship derived from experimental data:

$$
V_{d}=V \epsilon^{-0.5}\left(0.375 T+0.2274 T^{2}\right) .
$$

Finally, the evolution equation for droplet diameter is obtained in StarCD by solving

$$
\frac{d D_{d}}{d t}=-\frac{D-D_{m}}{\tau_{b}},
$$

where $\tau_{b}$ is the dimensional breakup time constant $\left(T D \epsilon^{0.5} / V\right)$. We will return to these time scales in the discussion of the droplet breakup behavior in both the tractor-trailer and tire simulations.

Quantitatively modeling spray impingment on walls is also computationally difficult. We have used the the model of Bai et al. (2002) implemented in StarCD to attempt to capture these effects in the simulations presented here. The Bai model was developed to model the impingement of gasoline spray on engine cylinder walls. The model includes a wide range of droplet-wall collision behaviors: sticking (dry walls only; we assume wetted walls in this work), spreading, rebounding and splashing. The two important non-dimensional numbers in the model are a modified Weber number using the wall- 


\begin{tabular}{lll}
\hline mode & $T$ & Weber range \\
\hline Vibrational & $6(W e-12)^{-0.25}$ & $12-18$ \\
Bag & $2.45(W e-12)^{0.25}$ & $18-45$ \\
Bag-and-stamen & $14.1(W e-12)^{-0.25}$ & $45-351$ \\
Sheet stripping & $0.766(W e-12)^{0.25}$ & $351-2670$ \\
Wave crest stripping & 5.5 & $>2670$ \\
\hline
\end{tabular}

TABle 1. Dimensionless total breakup time, $T$, for droplets as function of Weber number from Pilch \& Erdman (1987). Note that the Pilch and Erdman paper has a sign error in the relationship for the bag-and-stamen mode $T$ that is clearly evident upon comparison with their figure illustrating the relationship between $T$ and We.

\begin{tabular}{ll}
\hline Mode & modified Weber range \\
\hline Rebound & $5<W e_{d}$ \\
Spread & $5<W e_{d}<1320 L a^{-0.18}$ \\
Splash & $W e_{d}>1320 L a^{-0.18}$ \\
\hline
\end{tabular}

TABLE 2. Droplet wall impingement regimes from Bai et al. (2002).

normal velocity magnitude, $W e_{d}$, and the Laplace number,

$$
L a=\frac{\rho \sigma D}{\mu^{2}},
$$

which characterizes the ratio of inertial to surface tension forces in the droplet. Based on empirical fitting of experimental data, Bai et al. (2002) obtained the relationships in Table 2 as a function of modified Weber number. With the impact type determined, the post-impingement characteristics are obtained. For the stick or spread modes, the droplets adhere to the wall; for the latter, the tangential velocity relative to the wall is modified. For a rebound collision, both the normal and tangential velocities are multiplied by an empirical restitution coefficient (which is negative in the case of the wall-normal velocity component) dependent on the incidence angle of the incoming velocity with the wall.

The case of splash is the most interesting: in this case, new computational particles are created (breakup events typically do not lead to this outcome in StarCD's parcel methodology). Two daughter droplets are created and some portion of the original drop remains stuck to the wall. The daughter or secondary parcels represent an equal amount of mass, with the total mass of both determined by an empirical, randomly specified ratio of the total secondary to incident droplet mass ratio:

$$
r_{s}=0.2+0.9 X_{r},
$$

where $X_{r}$ is a uniform deviate random number between 0 and 1 . The mass of the fluid remaining stuck to the wall is simply $\left(1-r_{s}\right) m_{d}$, where $m_{d}$ is the mass of the incident 
droplet (parcel). As each parcel represents a fixed mass of particles, the size and number of droplets in each secondary parcel are calculated using a mass balance and a size randomly selected within an appropriate range defined by the modified Weber and Laplace numbers. Note that StarCD uses an older implementation of the Bai model; the cited reference incorporates size distribution data into this process. Estimates for the secondary droplet ejection angle are obtained by randomly selecting one secondary droplet ejection angle in the range of $5^{\circ}-50^{\circ}$ and then obtaining the remaining daughter droplet angle from conservation of tangential momentum. Lastly, the velocity magnitude is obtained by invoking energy conservation in combination with an experimental size-velocity correlation.

Finally, we note that the effects of droplet coalescence and turbulent dispersion are accounted for in our simulations. The former are modeled using a StarCD-specific implementation that is based on a statistical approach that does not lead to the generation of new parcels; instead the mass and number of droplets represented by a computational parcel is modified if and when collisions occur. The turbulent dispersion model is used to account for the well-known shortcoming that RANS simulations do not account for the interaction of particles or droplets with small flow features, giving rise to erroneous disperion estimates (Apte et al. 2003). Using this model attempts to rectify this problem by adding an $a d$ hoc approach that adds a random fluctuation velocity proportional to the local turbulent kinetic energy to the droplet velocity, which would otherwise be proportional to the local ensemble average velocity. Finite inertia effects are also accounted for in the response of the droplets to these fluctuations. However, in scoping tests in a cross-flow atomization problem we found that even with this model, droplet dispersion was underestimated.

\subsection{Mesh generation}

The starting point for the analysis was a surface scan of the actual Generic Conventional Model (GCM) 1:8 scale model used in wind tunnel tests at NASA-Ames Research Center (Salari et al. 2004b). A surface scan was used since available CAD data was unsuitable for CFD problem definition. For the computations, full-scale dimensions were used; the overall length is $20.16 \mathrm{~m}$, the gap between the tractor and trailer is $1.06 \mathrm{~m}$, and the offset of the model from the ground is $0.075 \mathrm{~m}$. Since the surface scan includes imperfections in the the model surfaces such as pressure taps as well as warpage in the model due to bending around the wind tunnel mounting posts, some care was required in the mesh generation process.

The GCM mesh for both the baseline and base flap cases was generated using the StarCD automotive meshing package es-aero. This tool utilizes a surface wrapper based approach combined with a hexahedral-dominant far-field mesh. The surface wrapper minimizes issues with surface flaws or imperfections by creating a "wrapped" surface that is smooth and continuous. Once the surface is "wrapped" a boundary layer extrusion is generated and transitioned to the outer cartesian mesh using trimmed hexahedral elements. Refinement for boundary layers near the ground and for arbitrary regions in the wake can also be specified. For the purposes of this study, a moving ground plane was used but the wheels of the GCM were separated by a small distance $(0.075 \mathrm{~m})$ for ease of meshing. A local cylindrical coordinate system was used at each wheel to impose a rotational boundary condition consistent with the ground and free stream velocities.

The parameters used in the mesh generation within es-aero, which were identical for both the baseline and drag-reduced case, are presented in Table 3. A schematic of the geometry and a snapshot of the mesh are given in Figure 1. The total mesh size is approximately 1.5 million cells; checks on $y^{+}$for the height of the first cell off the wall 


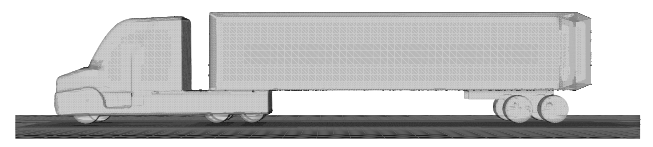

$\underset{-\mathrm{Y}}{\mathrm{z}} \mathrm{x}$

FIGURE 1. Geometry and mesh of GCM tractor-trailer model (with base flaps). Left:
geometry; Right: mesh as seen from side.

FiguRE 1. Geometry and mesh of GCM tractor-trailer model (
geometry; Right: mesh as seen from side.

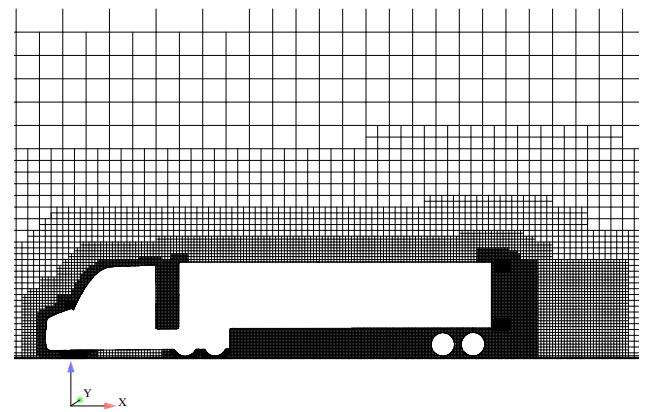

\begin{tabular}{ll}
\hline Parameter & Setting \\
\hline Streamwise domain size (in vehicle lengths) & $-2<X<3$ \\
Spanwise domain size (mm) & \pm 12912 \\
Vertical domain size (vehicle heights) & 4 \\
Ground offset distance (mm) & 25.4 \\
Surface wrapper preferred cell size/max cell size (mm) & $128 / 512$ \\
Extrusion layer total thickness (mm) & $10.8 \mathrm{~mm}$ \\
Cell size at wall (mm) & $3.6 \mathrm{~mm}$ \\
Boundary layer cell layers & 3 \\
Wake refinement box length (vehicle lengths)/cell size $(\mathrm{mm})$ & $0.4 / 64$ \\
Outer cartesian mesh cell size: preferred/min/max $(\mathrm{mm})$ & $128 / 32 / 2048$ \\
Ground refinement region height (mm) & 200 \\
Thickness of first cell on ground plane (mm) & 2.2 \\
\hline
\end{tabular}

TABLE 3. Settings for es-aero mesh generation for GCM study. Note that specific cell sets on the corners of the tractor and trailer were meshed using specific wrapper and nearby volume resolutions.

showed that values were between 2 and 250 with a mean of 45 , which is appropriate for the wall function approach used here. This coarse resolution was used to allow quick turnaround of the preliminary simulations presented here. The effects of mesh resolution using StarCD/es-aero for tractor-trailer aerodynamic problems are discussed in Pointer (2004); solutions obtained using coarse resolutions such as those used here exhibit the correct qualitative trends but are not quantitatively correct for metrics such as pressure distribution. Since we are primarily concerned with the interaction of a dispersed spray with large-scale flow features downstream of the tractor-trailer, we anticipate that the results presented here would be qualitatively unaffected by using finer mesh resolutions.

\subsection{Problem definition in StarCD}

Standard inflow and outflow boundaries were used at the streamwise inlet and outlet faces respectively. A uniform velocity of $29 \mathrm{~m} / \mathrm{s}$ (approximately $65 \mathrm{mph}$ ) was specified at the inlet. A moving ground plane with velocity equal to the inlet velocity was also defined. Wheel rotation is specified by defining a local coordinate system at the wheel hub; all cell faces corresponding to the wheel are associated with this local coordinate 


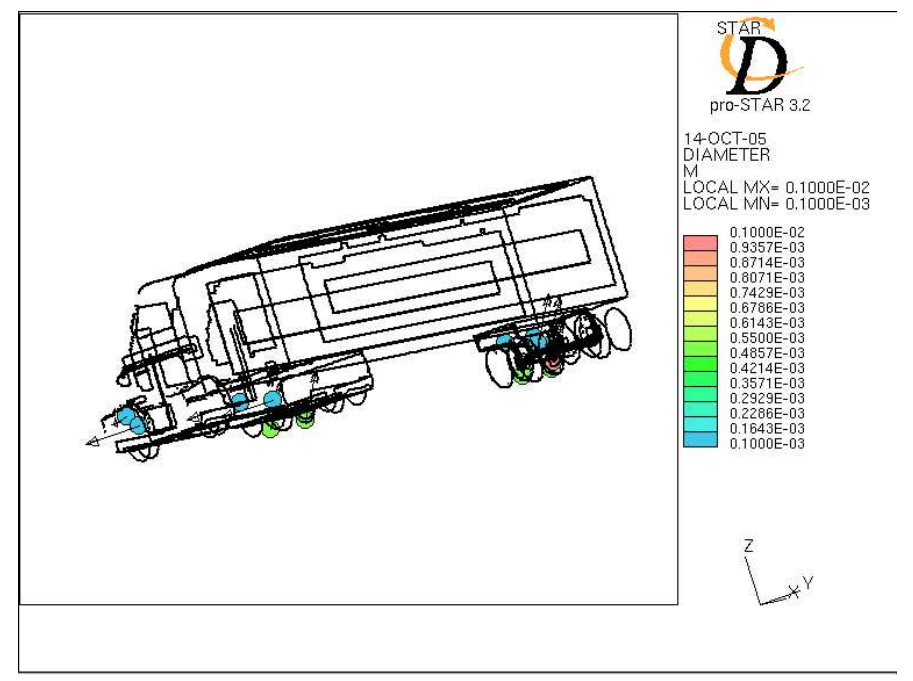

Figure 2. (Color) Snapshot from StarCD showing initial droplet size and velocity vectors for GCM spray dispersion tests.

system and a rotation rate giving a tangential velocity equal to the ground plane velocity is specified.

The spatial discretization scheme used in the simulations was StarCD's proprietary Monotone Advection and Reconstruction Scheme (MARS) with a blending factor of 0.5 for both the velocity components and the pressure. The "Pressure Implicit with Splitting Operators" (PISO) scheme (Issa 1986), which is a time-implicit numerical integration scheme, was used with a pressure underrelaxation factor of 0.1 . We attempted to take use the largest possible timestep that would still achieve numerical convergence and stability. With a timestep of 0.001 seconds, the average Courant number was approximately unity with the maximum Courant number in the domain typically being an order of magnitude greater.

The droplet injection parameters were challenging to specify since there are no experimental data available. There are many possible "injection" modes: tire tread water ejection, aerodynamic stripping of water sheets in the tire treads, droplet "flinging" from the treads (much like the water being thrown from a bicycle wheel onto a rider's back), and droplet creation from jets or large drops hitting the inside surfaces of wheel wells or the underside of the tractor-trailer. Size distributions and characteristic velocities for these modes are completely unknown as full scale tractor-trailer testing of these detailed physics is nearly impossible. In collaboration with Prof. Fred Browand at USC, we have started to experimentally investigate the breakup processes of water jets ejected from tire treads in a controlled environment (McCallen 2005). For this study we have assumed that the water from the tire treads is ejected at a $45^{\circ}$ angle to the ground at a velocity equal the ground speed. We have approximated wheel well and undersurface collisions on the GCM tractor (which does not include detailed wheel models) by using injectors at a $30^{\circ}$ angle to the flow and a velocity roughly twice that of the free stream to account for the wheel well aerodynamics. We have used a variety of droplet sizes, ranging from 0.01 to $1 \mathrm{~mm}$ since detailed droplet size distributions are unknown. The injection locations, sizes and velocities are shown in Figure 2. We have assumed a mass flowrate of $4.5 \mathrm{~kg} / \mathrm{s}$ based on a rough estimate of the volume of water displaced by a tire tread from the road at each injection point and injected 200 parcels/second into the computational domain. 

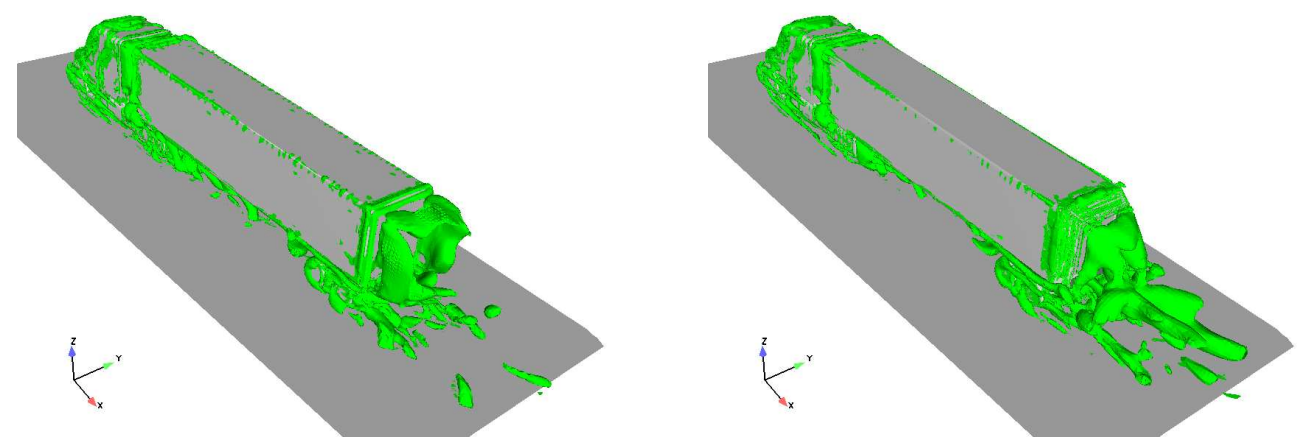

FiguRE 3. (Color) Instantaneous flow visualization of vortex structures in GCM wake using isosurfaces of $\mathrm{Q}(\mathrm{Q}=100)$. Left: Baseline; Right: Base Flaps. Note extended tubelike vortex structures in the base flap case.

The simulations used a statistically converged unsteady RANS simulation of the GCM and then reset the timing with the injection of droplets. A timestep of 0.001 seconds was used and the total time period considered for these simulations was 1.5 seconds, representing a droplet travel distance of roughly 45 meters or 2 vehicle lengths at free stream velocity. The runs reported here required between 40 to 60 Pentium 4 processors on the LLNL MCR cluster and took approximately 40 seconds wall clock time per computational time step, for a total simulation time of roughly 20 hours for each case including file operations.

\section{Results}

\subsection{Effect of add-on device (base flaps) on spray dispersion near tractor-trailers}

In this section, we demonstrate that the modification of the tractor-trailer flow field due to a drag-reducing device can lead to substantial changes in the transport of spray. Base flaps are a promising drag-reducing add-on device, with drag reductions of $10 \%$ shown in both computational and experimental studies. We first consider how base flaps modify the flow field and then examine the differences in the spray behavior.

In Figure 3 we present instantaneous snapshots of the vortical structures in the wake using isosurfaces of the second invariant of the velocity gradient tensor, Q:

$$
Q=\frac{1}{2}\left([\operatorname{tr}(\nabla u)]^{2}-\operatorname{tr}\left[(\nabla u)^{2}\right]\right)
$$

where $\nabla u$ is the velocity gradient tensor. As shown by Blackburn et al. (1996) and Dubief $\&$ Delcayre (2000), the isosurfaces of the positive values of Q correspond to regions in the flow where rotation is greater than extension and identify coherent vortex structures. The modifications to the wake structure are subtle and dynamic in nature; however, the largest difference is that the addition of base flaps causes a strong "downwash" in the wake. This downward flow stengthens the two trailing vortex structures that are attached to the bottom corners of the trailer as seen in the figure. An alternative visualization of this effect is shown in Figure 4, where streamlines in the centerline plane of the GCM are presented for both the baseline and base flap cases. The strong downward flow in the base flap case is clearly evident.

We anticipate that the strong downwash generated by the base flaps will negatively impact the spray transport by focusing the spray into the space occupied by a following or passing motorist. As shown in Figure 5, this is indeed the case: droplets are clearly 

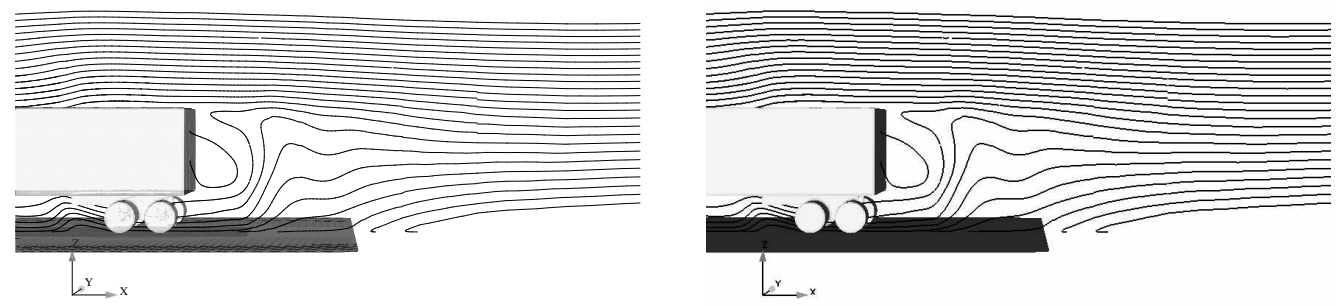

FigURE 4. Instantaneous flow visualization of streamlines in center plane of GCM wake. Left: Baseline; Right: Base Flaps. Note that the flow is directed more strongly towards the ground in the base flap case.
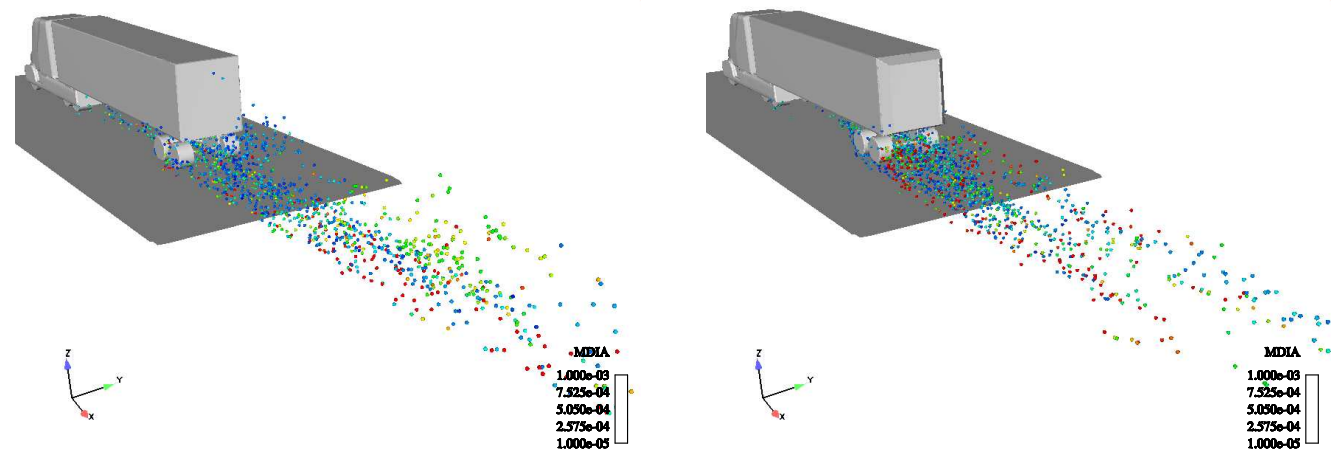

Figure 5. (Color) Instantaneous visualization of spray in GCM wake. Left: Baseline; Right: Base Flaps. Note that the spray is more tightly contained in the trailing vortex region in the base flap case; animations clearly show the droplets rotating about the vortex core.

"focused" into the stronger vortex structure present in the base flap case. Droplets injected into the domain from the tractor are sucked underneath the trailer in both cases, but without the base flaps the droplets are actually transported to the opposite side of the vehicle creating a more diffuse spray cloud. The differences in dispersion can be quantified by considering the spray cloud properties in a volume representative of the following and passing zone occupied by a motorist.

In all of the discussion that follows, it should be emphasized that the spray plume properties are a strong function of the injection parameters: size, velocity and injection rate, which were specified using "best guess" estimates. It is critical that experimental data, currently being obtained by Fred Browand, be included in the boundary conditions to obtain a more realistic visibility reduction estimate than the semi-quantitative results obtained here. With this caveat in mind, a number of quantitative metrics are used to describe the spray plume behind the tractor-trailer:

- Number density and size distribution

- Dispersion distance from ground or centerline

- Estimated visibility using concentration maps

We consider all of these metrics using an instantaneous visualization at a fixed time (1.0 seconds) in both simulations. It would be straightforward to extend these measurements to a series of snapshots in time and average the data; this analysis has not been performed here, but would be a close analog to the tests of Dumas \& Lemay (2004). 


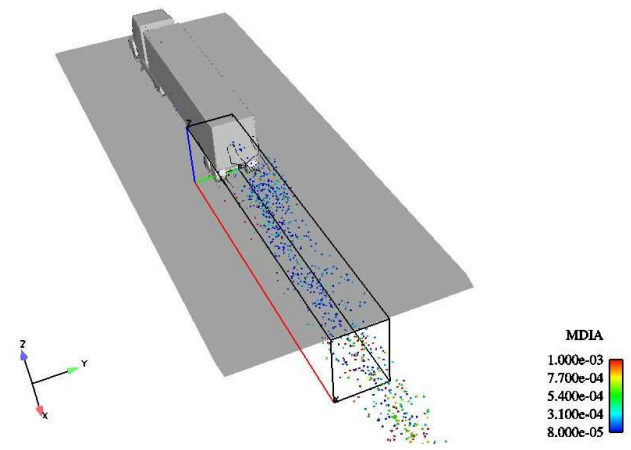

FiguRE 6. Sampling box for spray statistics calculations. Only particles contained in this box, representative of the passing zone for a motorist, are considered. Box coordinates in meters $(\mathrm{x}, \mathrm{y}, \mathrm{z}):[20: 39,-2.5: 2.5,0.5: 3.8]$
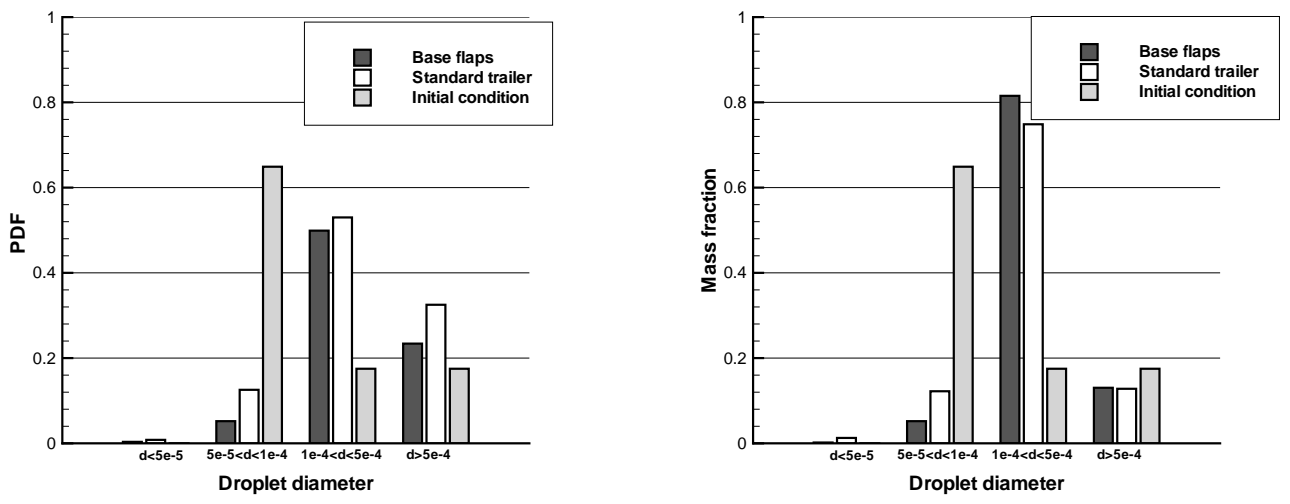

FiguRE 7. Size (left) and mass (right) distributions of spray at start of injection and in sampling box at $\mathrm{t}=1.0 \mathrm{sec}$

\subsubsection{Concentration and size distribution}

The simplest metric is a measurement of the concentration of particles in a fixed sampling domain; here we consider a rectangular box behind and to the left of the tractortrailer in the range $(x, y, z) \in[20: 39,-2.5: 2.5,0.5: 3.8]$ that roughly corresponds to the "passing zone" behind and to the left of the vehicle. A picture of the sampling domain is shown in Figure 6. The number density of parcels in the drag-reduced case is approximately $50 \%$ greater than in the baseline case, with the the standard GCM having having 2.15 parcels $/ \mathrm{m}^{3}$ and the base flap case 3.4 parcels $/ \mathrm{m}^{3}$. Note that each parcel represents a fixed mass of water of $22.5 \mathrm{~g}$, so the modest differences in number of parcels corresponds to a large difference in the number of "real" droplets. For example, using a droplet diameter of $1 \times 10^{-4} \mathrm{~m}$, a difference in parcel density of 1.25 parcels $/ \mathrm{m}^{3}$ corresponds to an additional 850,000 droplets $/ \mathrm{m}^{3}$. This observation supports the visual observation that the spray is less dispersed with the base flaps in place.

Figure 7 provides information on the size and mass distribution of the spray cloud in the sampling box. Compared to the initial size distribution, the resulting spray has a bias towards slightly larger drops in the range of $1 \times 10^{-4}$ to $5 \times 10^{-4} \mathrm{~m}$ both in terms of the mass and size distributions. This result suggests that coalescence may be 


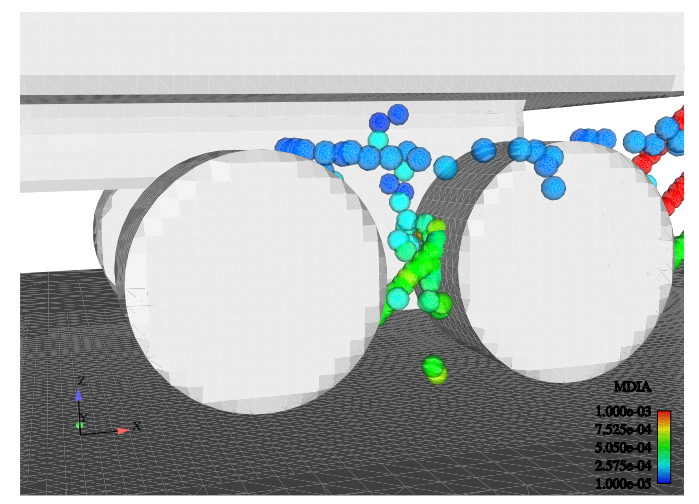

FigURE 8. Snapshot from StarCD showing collision-induced breakup on the rear tire of the trailer tandem tire assembly. The "jets" of injected droplets are hitting the tire, with parcels created for the sticking and breakup processes.

important and that if any breakup is occurring, it involves only the largest drops in the size range $\left(D>5 \times 10^{-4} \mathrm{~m}\right)$. Collisional coalescence events are seen on the back tires as shown in Figure 8. The resulting drops that "spill" off of the tire appear to be larger than those impacting the tire; examining StarCD's output files there are a modest number of coalescence events reported (typically 10-50) during each 0.2 second simulation period.

Aerodynamic breakups are difficult to observe since the parcels do not break, but only change color in the visualizations. A more quantitative estimate can be obtained by examining the time history of a particular parcel's droplet count (viewable within StarCD) and by exmaining the parcel Weber number to obtain the relevant breakup time. We have found that no aerodynamic breakup events are reported over the time of the simulation. To understand why, we can consider the properties of a droplet with diameter $1 \times 10^{-3} \mathrm{~m}$. Using the properties of water and a representative slip velocity of 15 $\mathrm{m} / \mathrm{s}$, the Weber number is 3.7. The empirical droplet breakup model used here predicts no breakup for $W e<12$; reducing the diameter simply makes the Weber number smaller. For a larger droplet with a diameter of $1 \mathrm{~cm}, W e=38$ and the breakup time is $\approx 0.1$ seconds. A simple ballistic trajectory from the bottom of the tire to the underside of the tractor-trailer takes a comparable amount of time, meaning that aerodynamic breakup of a drop once separated from the initial jet ejected from the tire tread or splash is unlikely. We can conclude that droplet breakup and coalescence due to collisions is critical to an accurate modeling of tractor-trailer spray.

\subsubsection{Dispersion estimation}

To quantify the amount of dispersion, we choose to use the r.m.s. distance from the centerline of all droplets in the sampling box shown in Figure 6. The horizontal dispersion is shown in Figure 9. The addition of baseflaps gives both larger dispersion and larger variations in dispersion of the smallest and largest drops compared to the standard GCM. As seen in the instantaneous visualization, particles of all sizes interact with the trailing vortex created by the base flaps and are pushed farther to the side of the tractor-trailer. The smallest drops are concentrated in the core of this vortex, while the largest drops are entrained and focused on the outside of the vortex. It would appear that drops of intermediate size behave on average in a similar manner. 

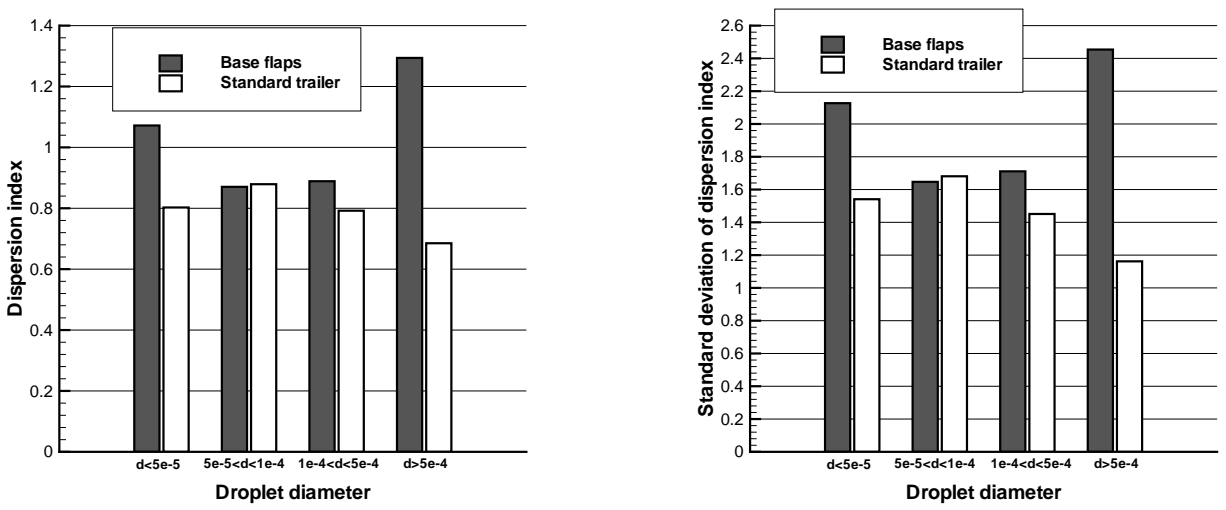

Figure 9. R.m.s distance from ground plane (left) and standard deviation (right) of spray dispersion index distributions in sampling box at $\mathrm{t}=1.0$ seconds.

\subsubsection{Visibility reduction by spray}

Finally, we consider the reduction in motorist visibility by the spray plume in both cases. The reduction in visibility is related to the concentration of the droplets in the plume. Here, we consider a sampling box from the rear of the trailer to $20 \mathrm{~m}$ downstream, spanwise extent $y \in(-2.5,2.5)$ and height $z \in(0.5,4.0)$. Within this box we calculate the concentration in sampling boxes that have a specified $\Delta y$ and $\Delta z$ and extend the entire length of the domain. The concentration can be related to the visibility through an empirical relationship, based on the Beer-Lambert law, relating the visibility in meters, $\psi$ to an extinction coefficient, $\beta$ (Kunkel 1983):

$$
\psi=-\frac{\ln (0.02)}{\beta} \text {. }
$$

Experimentally obtained values of the extinction coefficent are available for both fog and rain; we have used the values for rain in Rensch \& Long (1970). Rensch \& Long (1970) provide the extinction coefficient as a function of rainfall rate in $\mathrm{mm} /$ hour. To convert the spray parameters from the simulations to this quantity, the mass fraction of each subdomain is multiplied by the total mass flux through the entire sampling box. The effective rainfall rate is then obtained by dividing the resulting volume flowrate by the area of the box and a value of $\beta$ obtained from a curve fit of the data in Rensch \& Long (1970). The resulting visibility calculations are shown in Figure 10.

The results show that the region of poor visibility, which we define as less than $100 \mathrm{~m}$, is shifted to the left and up when baseflaps are added.

This suggests that following the tractor-trailer, visibility might be better but that passing may be made more difficult as spray is pushed into the windshield area assuming a passing vehicle height of about $1.5 \mathrm{~m}$. The maximum visibility reduction is approximately $90 \%$, which correlates well with the experimental data of Dumas \& Lemay (2004). However, the results also show significantly less visibility (effectively none) reduction at distances greater than $1 \mathrm{~m}$ left of the tractor-trailer than in the study of Dumas \& Lemay (2004) and this is likely the result of the choice of inlet conditions. This result illustrates that visibility may be impaired by the use of trailer-mounted base flaps. However, to be certain of the absolute reductions in visibility, better experimental data for spray inlet conditions is required. We speculate that the removal of the bottom plate in the baseflap assembly might act to reduce the "updraft" of water spray which might improve visi- 

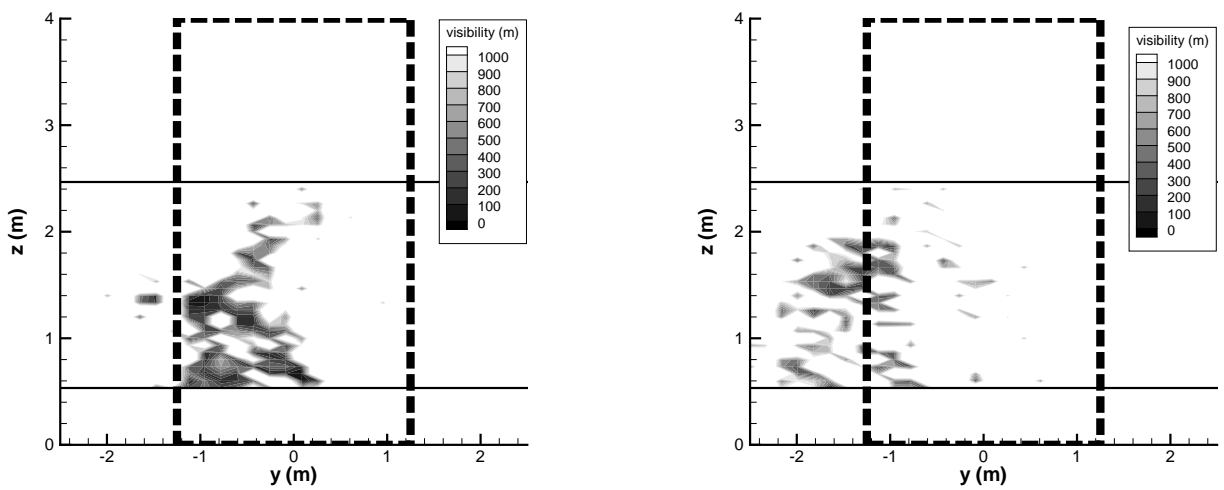

FiguRE 10. Estimated visibility distance through spray plume in from $20 \mathrm{~m}$ behind tractor-trailer for case of standard GCM (left) and base flaps (right). The total height of the tractor-trailer, including the wheels, is defined by the dotted lines. A typical car windshield is around $1.5 \mathrm{~m}$ above ground.

bility. We also note that other devices such as underbody wedges or side skirts that act to create low pressure regions underneath the trailer might actually act to improve the visibility by pulling drops underneath the truck.

\section{Conclusions and suggestions for future work}

A preliminary investigation of spray transport in heavy vehicle and tire wakes was completed using the commercial code StarCD. The empirical spray models included collision and aerodynamic breakup modes; the former was found to be of primary importance. The central findings of this study are:

- CFD codes and computational resources are now capable of of investigating the splash and spray problem

- Addition of base flaps may actually act to decrease motorist visibility by focusing droplets in the passing zone behind and to the left of the tractor-trailer

- Based on this result, we speculate that other aerodynamic drag-reducing add-on devices such as side skirts or belly boxes may actually act to improve visibility by "sucking" spray underneath the vehicle

This first attempt at simulating spray dispersion in tractor-trailer or tire wakes employed a number of simplifications and assumptions. Some important details that require further study are:

- Droplet sizes and velocities as well as injection points were assumed. The work of Fred Browand at USC will improve the specification of these considerably

- The breakup model for droplet-surface collisions is designed for gas in engine cylinders. An experimental study examining the breakup physics or simply to obtain the correct empirical coefficients for the model used would improve the accuracy

- The effect of crosswinds on devices is clearly important and is probably responsible for much of the apparently contradictory experimental data on spray reduction. This effect is straightforward but computationally expensive to obtain as the mesh resolution requirements are severe

- The most accurate method to simulate the combined splash and spray problem is to couple the free-surface type models required for splash with the Lagrangian particle- 
tracking type models used for spray; this coupling is quite difficult to implement accurately and requires efficient means of capturing breakup

- Unsteady RANS has well-known shortcomings for separated bluff-body wake flows; computationally efficient LES or hybrid RANS-LES models are necessary to improve to temporal accuracy and spatial resolution of vortical structures in the flow that are known to strongly affect particle dispersion

In closing, although considerable empiricism is still required, CFD makes it possible to complete controlled studies of the effectiveness of spray suppression devices and may make the intelligent design of such devices a reality.

\section{Acknowledgments}

Helpful conversations with and feedback on this manuscript from Rose McCallen, Kambiz Salari, Jason Ortega and Paul Castellucci is gratefully acknowledged. This work was performed under the auspices of the U.S. Department of Energy by the University of California, Lawrence Livermore National Laboratory under Contract No. W-7405-ENG48

\section{REFERENCES}

CD ADAPCO 2005 StarCD v.3.24, http://www.cd-adapco.com/products/STARCD/index.html.

Allan, J. \& Lilley, G. 1983 The reduction of water spray from heavy road vehicles. Int. J. of Vehicle Design SP3, 270-307.

Apte, S., Mahesh, K., Moin, P. \& Oefelein, J. 2003 Large-eddy simulation of swirling particle-laden flows in a coaxial-jet combustor. Intl. J. Multiphase Flow 29, 1311-1331.

Bai, C., Rusche, H. \& Gosman, A. 2002 Modeling of gasoline spray impingment. Atomization and Sprays 12, 1-27.

Blackburn, H., Mansour, N. \& Cantwell, B. 1996 Topology of fine scale motions in turbulent channel flow. J. Fluid Mech. 310, 269-292.

Bussman, M., Mostaghimi, J. \& Chandra, S. 1999 On a three-dimensional volume tracking model of droplet impact. Phys. Fluids 11 (6), 1406-1417.

Chen, M., Kontomaris, K. \& McLaughlin, J. 1998 Direct numerical simulation of droplet collisions in a turbulent channel flow. part i:collision algorithm. Int.J.Multiphase Flow 24, 1079-1103.

Crowe, C., Sommerfeld, M. \& Tsuji, Y. 1998 Multiphase flows with droplets and particles. CRC Press.

Dubief, Y. \& Delcayre, F. 2000 On coherent-vortex identification in turbulence. J. Turbulence 1, 011 .

Dumas, G. \& Lemay, J. 2004 Splash and spray measurement and control: Recent progress in quebec. In The Aerodynamics of Heavy Vehicles: Trucks, Buses and Trains (ed. R. McCallen, F. Browand \& J. Ross), Lecture Notes in Applied and Computational Mechanics, vol. 19, pp. 533-547. Springer.

Durbin, P. \& Reif, B. P. 2003 Statistical Theory and Modeling for turbulent flows. Wiley.

Goering, E. \& Kramer, W. 1987 a Improving the active and passive safety of commercial vehicles by lateral chassis fairings. Automobiltech Zeitscrhift 89, 659-666, in German.

Goering, E. \& KRAmer, W. $1987 b$ Lateral chassis fairings for commercial vehicles: An effective aid for the further improvement for aerodynamics, safety, and environmental acceptability. Automobiltech Zeitscrhift 89, 481-488, in German.

IssA, R. 1986 Solution of implicitly discretized fluid flow equations by operator splitting. $J$. Comp. Phys. 62, 40-65.

Josserand, C. \& Zaleski, S. 2003 Droplet splashing on a thin liquid film. Phys. Fluids 15 (6), $1650-1657$.

Kolev, N. 1993 Fragmentation and coalescence dynamics in multiphase flows. Exptl. Thermal and Fluid Sci. 6, 211-251. 
Kunkel, B. 1983 Parameterization of droplet terminal velocity and extinction coefficient in fog models. J. Climate and Appl. Meteorology 23, 34-41.

Lasheras, J., Eastwood, C., Martinez-Bazan, C. \& J.Montanes 2002 A review of statistical models for the break-up of an immiscible fluid immersed into a fully developed turbulent flow. Int.J.Multiphase Flow 28, 274-278.

Lasheras, J. \& Hopfinger, E. 2000 Liquid jet instability and atomization in a coaxial gas stream. Ann. Rev. Fluid Mech. 32, 275-308.

Lin, S. \& Reitz, R. 1998 Drop and spray formation from a liquid jet. Ann. Rev. Fluid Mech. 30, 85-105.

Manser, M., Koppa, R. \& Mousley, P. 2003 Evaluation of splash and spray suppression devices on large trucks during wet weather. Tech. Rep.. AAA Foundation for Traffic Safety, www.aaafoundation.org.

MCCALLEn, R. 2005 May 2005 working group meeting on heavy vehicle drag. Tech. Rep. UCRLTR-214683. LLNL, http://eed.llnl.gov/aerodrag/publications.php.

Menter, F. 1994 Two-equation eddy-viscosity turbulence models for engineering applications. AIAA J. 32, 1598-1605.

NHTSA 2000 Update on the status of splash and spray suppression technology for large trucks. Report to Congress.

O.D.O.T. 2002 MCTD Form 2351A: Splash and Spray Suppressant Device Requirements, http://www.odot.state.or.us/forms/motcarr/2351a.pdf.

Pilch, M. \& ERdman, C. 1987 Use of breakup time data and velocity history data to predict the maximum size of stable fragments for acceleration-induced breakup of a liquid drop. Int.J.Multiphase Flow 13 (6), 741-757.

Pointer, W. 2004 Evaluation of commercial cfd code capabilities for prediction of heavy vehicle drag coefficients. Tech. Rep.. AIAA-2004-2254.

Rensch, D. \& Long, R. 1970 Comparative studies of extinction and backscattering by aerosols, fog and rain at $10.6 \mu$ and $0.63 \mu$. Applied Optics 9 (7), 1563-1573.

Salari, K., Ortega, J. \& Castellucci, P. $2004 a$ Computational prediction of aerodynamic forces for a simplified integrated tractor-trailer geometry. Tech. Rep.. AIAA-2004-2253.

Salari, K., Ortega, J. \& Castellucci, P. $2004 b$ A study of reynolds number effects and drag-reduction concepts on generic tractor-trailer. Tech. Rep.. AIAA-2004-2251.

SCARDOVElli, R. \& ZALESKI, S. 1999 Direct numerical simulation of free-surface and interfacial flow. Ann. Rev. Fluid Mech. 31, 567-603.

Sethian, J. \& Smereka, P. 2003 Level set methods for fluid interfaces. Ann. Rev. Fluid Mech. 35, 341-372.

Sundaram, S. \& Collins, L. 1997 Collision statistics in an isotropic particle-laden turbulent suspension. part i: Direct numerical simulations. J. Fluid Mech. 335, 75-109.

Tang, L., Wen, F., Yang, Y., Crowe, C., Chung, J. \& Troutt, T. 1992 Self-organizing particle dispersion mechanism in a plane wake. Phys. Fluids A 4 (10), 2244-2251.

Tsouris, C. \& TAvlarides, L. 1994 Breakage and coalescence models for drops in turbulent dispersions. AIChE J. 40 (3), 395-406.

Weir, D., Strange, J. \& Heffley, R. 1978 Reduction of adverse aerodynamic effects of large trucks: Vol. 1: Technical report. Tech. Rep. FHWA-RD-79-84, STI-TR-1093-01. Systems Technology, Inc., Interim Report to Federal Highway Administration.

WiLcox, D. 2002 Turbulence Modeling for CFD. DCW Industries. 